\title{
The management of superficial venous incompetence
}

The third author of this clinical review by $\mathrm{P} B$ van den Boezem and colleagues (BMJ 2011;343:d4489, doi:10.1136/bmj.d4489) would like us to advise readers that his name is E G J M le Cocq

d'Armandville [not E le Cocq d'Armandville, as published]. 